$\begin{array}{cl}\begin{array}{cl}\text { Revue } \\ \text { de Ihistoire } \\ \text { des religions }\end{array} & \text { Revue de l'histoire des religions } \\ & \begin{array}{l}1 \mid 2010 \\ \text { Beauté du rite }\end{array}\end{array}$

Art et liturgie aujourd'hui : à propos de six récentes églises parisiennes (1997-2005)

Art and Liturgy Today: Six Recent Parisian Churches (1997-2005)

Isabelle Saint-Martin

\title{
OpenEdition
}

Édition électronique

URL : http://journals.openedition.org/rhr/7569

DOI : $10.4000 /$ rhr.7569

ISSN : 2105-2573

Éditeur

Armand Colin

Édition imprimée

Date de publication : 1 mars 2010

Pagination : 127-146

ISBN : 978-2200-92612-0

ISSN : 0035-1423

Référence électronique

Isabelle Saint-Martin, «Art et liturgie aujourd'hui : à propos de six récentes églises parisiennes

(1997-2005) », Revue de l'histoire des religions [En ligne], 1 | 2010, mis en ligne le 01 mars 2013,

consulté le 01 mai 2019. URL : http://journals.openedition.org/rhr/7569; DOI : 10.4000/rhr.7569

Tous droits réservés 


\section{Art et liturgie aujourd'hui : à propos de six récentes églises parisiennes (1997-2005)}

Entre 1997 et 2005, à l'initiative notamment de Mgr Lustiger, six nouvelles églises ont été achevées à Paris et à La Défense. Malgré la diversité des solutions architecturales (Architecture Studio, C. Callies et J.-M. Duthilleul, F. Hammoutène...), elles partagent un même objectif de visibilité dans l'espace urbain, sans monumentalité, et une grande attention portée à la conception de l'espace intérieur qui met en valeur une liturgie participative. Examiner les dispositifs esthétiques dans lequel le rite se déploie met en évidence l'importance attachée à la dimension symbolique de l'espace et des auvres avec le souci d'accompagner fidèle ou visiteur dans un cheminement spirituel en accord avec le sens de l'édifice et la spécificité du lieu de culte.

\section{Art and Liturgy Today : Six Recent Parisian Churches (1997-2005)}

Between 1997 and 2005, thanks notably to Mgr. Lustiger, six new churches were completed in Paris and in La Défense. In spite of the diversity of the architectural schemes (Architecture Studio, C. Callies and $J-M$ Duthilleul, F. Hammoutène...) they share the same goal of visibility within the urban space without trying to be monumental, and a specific attention given to the conception of inner space in order to emphasize a participative liturgy. If we study the aesthetics in which the rite takes place, we may point out the importance dedicated to the symbolic dimension of space and of works of art in order to assist the faithful or the visitor in a spiritual progression in accordance with the sense of the construction and the specificity of a place of worship. 
Lors de l'éloge du sculpteur qui a conçu le maitre-autel en bronze de Notre-Dame de Paris, Mgr Lustiger invitait à « montrer l'invisible sans le réduire au visible ${ }^{1} »$. Rapprocher ces mots des interrogations qui président à cette enquête collective sur les rapports entre liturgie et esthétique dans le christianisme incite à étudier quelques cas récents - six églises construites dans le diocèse de Paris ou sa proximité immédiate - qui offrent un corpus relativement homogène dans le temps, le lieu et les ambitions. La citation de Mgr Lustiger se réfère au thème classique du lien entre le visible et l'invisible (per visibilia ad invisibilia), mais une telle affirmation avait pu paraître perdre de sa pertinence en un temps où l'on prônait pour la modernité une forme d'ascèse visuelle. Une première lecture des suites du concile de Vatican II a parfois conduit à y déceler l'appel au dépouillement et à une sorte de carême esthétique. Réaffirmer l'intérêt de "montrer », de donner à voir, ouvre la voie à de nouvelles relations avec les artistes, sans toutefois négliger le risque d'une réduction à l'apparence. C'est au cœur de cette tension que l'on voudrait examiner non la liturgie catholique comme art dans son expression intrinsèque ${ }^{2}$, mais, dans une approche distincte, les dispositifs esthétiques dans lesquels elle prend place, qu'il s'agisse de la conception du sanctuaire, ou de l'intervention d'artistes dans la réalisation du mobilier liturgique comme dans l'installation spécifique d'œuvres d'art. Le cadre matériel dans lequel le rite se déploie entre en jeu dans la perception esthétique et sensorielle de celui-ci. S'agit-il seulement d'un décor - et l'on retrouverait alors les traces d'un débat pluriséculaire entre un souci du dénuement refusant toute concurrence visuelle avec l'essence de la cérémonie ou au contraire la notion d'accompagnement et d'honneur rendu au culte par la mise à contribution des sens - ou peut-on percevoir des liens plus étroits entre l'édifice et sa destination litur-

1. Paris Notre-Dame $(P N D), \mathrm{n}^{\circ} 1028,5$ février 2004, Mgr Lustiger, éloge de Jean Touret.

2. L'approche celle sera celle de l'histoire de l'art et de l'histoire religieuse et non celle d'un spécialiste de la liturgie. Sur ce point, voir notamment les travaux de Paul De Clerck, L'Intelligence de la liturgie, Paris, Le Cerf (1995), 2005; Jean-Yves Hameline, Une poétique du rituel, Paris, Le Cerf, 1997; François Cassingena-Trevedy, La Liturgie, art et métier, Genève, Ad Solem, 2007. 
gique $^{3}$ ? Situer le contexte du corpus retenu, puis examiner les dispositifs mis en place pour constituer des ensembles signifiants, permettra de préciser les interactions entre l'organisation de l'espace et l'action rituelle qui s'y déroule.

\section{SACRoSANCTUM CONCILIUM, ÉLÉMENT DE CONTEXTE}

Si rite et art, en particulier depuis l'affirmation d'une autonomie de la création artistique, sont deux notions distinctes, susceptibles d'entrer en concurrence dans leur mode d'expression symbolique ${ }^{4}$, c'est toutefois principalement dans la Constitution sur la liturgie que la mention de l'art apparait, dans les textes du Concile de Vatican $\mathrm{II}^{5}$. Sacrosanctum concilium place les « beaux-arts» «parmi les plus nobles activités de l'esprit humain» dont «l'art sacré est le sommet »; l'Église se proclame « amie des arts » et entend « requérir leur noble ministère ${ }^{6} »$ afin que les $«$ objets servant au culte soient vraiment dignes, harmonieux, beaux » (SC 122). Ce que reprend précisément La Présentation générale du missel romain (1969) sur l'aménagement des églises :

Les demeures sacrées et les objets destinés au culte divin seront vraiment dignes et beaux, capables de signifier et de symboliser les réalités surnaturelles. Par conséquent, l'église ne cesse de faire appel au noble ministère de l'art et elle admet les valeurs d'art de tous les peuples et de toutes les régions. (PGMR, $3^{\mathrm{e}}$ éd. 2002, chap. V, $\mathrm{n}^{\circ} 288-289$ ).

Cependant cet appel à l'art s'accompagne d'une notion de service qui l'associe étroitement à la finalité du lieu de culte ${ }^{7}$. Ainsi l'art de notre époque a «toute liberté de s'exercer pourvu qu'il serve les édifices et les rites sacrés avec le respect et l'honneur qui leur

3. Voir Jacques Vanuxem, «La querelle du luxe dans les églises après le concile de Trente », Revue de l'art, 1974, p. 48.

4. Albert Rouet, Art et liturgie, Paris, Desclée de Brouwer, 1992, p. 36.

5. Voir Sacrosanctum Concilium (abrégée SC), Le Concile Vatican II, 19621965 , texte latin et traduction française avec index et tables, préface par Giuseppe Alberigo, trad. par Raymond Winling, Paris, Le Cerf, 2003.

6. Voir les mêmes termes dans la Lettre aux artistes de Paul VI, 1964 et celle de Jean-Paul II, 1999.

7. Voir Antoine Bayer, «Des églises pour aujourd'hui. L'art sacré selon Vatican II », Études, juillet-août 1988, p. 85-93 ; François Boespflug, « Art et liturgie : l'art chrétien du XXI ${ }^{\mathrm{e}}$ siècle à la lumière de Sacrosanctum concilium », Revue des sciences religieuses, 78-2, 2004, p. 161-181. 
sont dus » (SC 123). Les recommandations s'inquiètent d'une possible prolifération du visuel et souhaitent réguler les dévotions particulières. Si « la pratique de proposer dans les églises des images sacrées à la vénération des fidèles » est maintenue selon les termes tridentins, il est précisé que celles-ci « seront exposées en nombre restreint et dans une juste disposition, pour ne pas éveiller l'étonnement du peuple chrétien et ne pas favoriser une dévotion mal réglée » (SC 125). Mais lorsque les pères du concile espéraient la «noble beauté plutôt que la seule somptuosité » (SC 124), le contexte social et artistique des années soixante fut surtout sensible à l'esprit de pauvreté et au refus du luxe. Le souvenir des années de guerre, puis le souci d'un refus du triomphalisme, non seulement dans le mode d'intervention sur la scène publique, mais encore dans le décor du sanctuaire, ont rencontré les aspirations d'une forme d'évangélisation qui, pour mieux transformer le monde de l'intérieur, cherchait, sur le modèle du « sel de la terre », l'enfouissement au détriment des formes de visibilité extérieure. Les arts visuels semblaient alors relever du superflu ou de l'ostentatoire. L'invitation au dépouillement et à la simplicité évangélique a parfois conduit à une perte du sens de l'art ou des objets anciens, certes antérieure aux années soixante, mais renforcée dans la période qui a suivi le concile ${ }^{8}$.

\section{UNE PRÉSENCE VISIBLE SANS ÊTRE IMPOSANTE}

L'architecture extérieure visait également à se fondre dans le paysage urbain ${ }^{9}$ et à tirer parti de locaux provisoires, salles polyvalentes, églises démontables... Mais les années 1980 voient réapparaître, et parfois rajouter sur des constructions récentes, des signes de visibilité tels qu'un clocher ou une grande croix apparente. La réflexion sur le retour du monumental ${ }^{10}$, que consacre la construction de la cathédrale d'Évry, croise la présence plus manifeste d'une pluralité

8. Voir Gilles Chazal, «L'art dans l'Église après Vatican II », Revue de l'art, 1974, p. 72-80.

9. Voir Franck Debié et Pierre Vérot, Urbanisme et art sacré. Une aventure $d u X X^{e}$ siècle, Paris, Criterion, 1990.

10. L'Architecture religieuse et le retour du monumental, actes des rencontres internationales d'Évry, 1989, EVREPY, 1990. 
religieuse sur le territoire français ainsi que les préoccupations du pontificat de Jean-Paul II et de la Nouvelle évangélisation. C'est dans ce contexte que Mgr Lustiger, archevêque de Paris en 1981, affirme la nécessité de construire plusieurs nouveaux lieux de culte dans la capitale. Alors que les réalisations récentes avaient surtout suivi le développement des banlieues ${ }^{11}$, Paris étant déjà riche de multiples églises, l'archevêché souligne l'importance des déplacements de populations au sein d'un quartier (place d'Alleray, $\mathrm{XV}^{\mathrm{e}}$ arrondissement ou Auteuil, XVI ${ }^{\mathrm{e}}$ ) ou l'émergence de nouvelles implantations (quartier Tolbiac et grande bibliothèque, XIII ${ }^{\mathrm{e}}$, quartier de l'Ourcq, XIX ${ }^{\mathrm{e}}$ ). Suivre ces évolutions par la création d'églises nouvelles entend rappeler qu'un lieu de culte n'est pas seulement l'expression de la piété des fidèles, mais aussi un pôle d'attraction apte à l'éveiller et à susciter une nouvelle fréquentation paroissiale. Parmi les réalisations récentes ${ }^{12}$, on retiendra ici plus particulièrement ${ }^{13}$ Notre-Dame-d'Espérance ${ }^{14}\left(\mathrm{XI}^{\mathrm{e}}\right.$, architecte Bruno Legrand, 1997, fig. 1 et 7), Notre-Dame-de-l'Arche-d'Alliance ${ }^{15}\left(\mathrm{XV}^{\mathrm{e}}\right.$, Martin Robain, Architecture Studio, 1998, fig. 2 et 8), Saint-Luc ${ }^{16}$ (XIX ${ }^{\mathrm{e}}$, architectes Pierre-Henri Montel et Christian Basset/BSST, 1999, fig. 3 et 9), la chapelle Notre-Dame-de-la-Sagesse ${ }^{17}$ (XIII $^{\mathrm{e}}$, architecte Pierre-Louis Faloci, 2000, fig. 4 et 10), Saint-François de Molitor $^{18}\left(\mathrm{XVI}^{\mathrm{e}}\right.$, architectes Jean-Marie Duthilleul et Corinne Calli-

11. Antoine Le Bas, Des sanctuaires hors les murs, églises de la proche banlieue parisienne, Paris, Éd. du Patrimoine, 2003.

12. Pour d'autres exemples voir L'Architecture d'aujourd'hui, «Sacré/Profane », 2004; Technique et architecture, $\mathrm{n}^{\circ}$ spécial «Espaces sacrés », $\mathrm{n}^{\circ} 459$, 2002.

13. Nous mentionnons le numéro de l'arrondissement, le nom de l'architecte, la date de la consécration. Les clichés accompagnant la présente étude sont de l'auteur, qui remercie Messieurs les curés de ces églises pour leur autorisation (pour une iconographie plus abondante, se reporter au site internet du diocèse de Paris : www.catholique-paris.com; et pour chaque édifice aux sites dont nous indiquons les adresses dans les notes ci-dessous).

14. Sur le site d'une église à reconstruire, 47 rue de la Roquette, 75011. Voir http://notredameesperance.free.fr; http://www.bla.fr/partenaires.html.

15. 81 rue d'Alleray, 75015. Voir Architecture Studio, Notre-Dame-de-l'Arched'Alliance, préface du Card. Lustiger, postface du P. Schwab, textes de F. Boyer, Paris, 1999. Voir http://www.ndarche.org; http://www.architecture-studio.fr.

16. 80 rue de l'Ourcq, 75019. Voir http://www.saintlucparis.com.

17. 13 bis, rue Abel-Gance, 75013, rattachée à la paroisse Notre-Damede-la-Gare. Voir http://monsite.wanadoo.fr//nd-lasagesse.fr; http://www. pierrelouisfaloci.com.

18. 44, rue Molitor, 75016. Voir http://www.saintfrancois-molitor.cef.fr. 
es, 2005, fig. 5 et 11), auxquelles il faut ajouter le cas particulier de la " Maison d'Église », Notre-Dame-de-Pentecôte sur l'esplanade de La Défense (Franck Hammoutène, 2001, fig. 6) selon le souhait de Mgr Favreau, archevêque de Nanterre, de marquer une présence chrétienne dans ce quartier des affaires qui prolonge l'ouest pari$\operatorname{sien}^{19}$. Chacun de ces édifices, souvent confiés à des architectes de grand renom, mériterait une approche monographique détaillée ${ }^{20}$. Loin de toute exhaustivité, un survol des principaux éléments voudrait faire apparaître, à travers les points communs comme les divergences, une possible ligne directrice pour les relations entre art et liturgie. Ces six églises, achevées entre 1997 et 2005, partagent une taille relativement modeste et une même forme de rapport à l'espace urbain environnant. Elles manifestent une visibilité retrouvée marquée par la présence de clochers, de façades travaillées ou de couleur, ainsi qu'une identité architecturale forte, bien que chaque fois différente, la plus spectaculaire étant sans doute le cube posé sur pilotis et entouré d'une résille métallique de Notre-Dame de l'Arche-d'Alliance (fig. 8). Toutefois, la façade de marbre de Saint-François de Molitor ou le mur-rideau en verre et aluminium de Saint-Luc, le mur entièrement calligraphié de Notre-Damed'Espérance, la brique rouge et les formes géométriques de NotreDame-de-la-Sagesse montrent qu'il n'y a pas ici un modèle type d'église du XXI ${ }^{\mathrm{e}}$ siècle, mais une volonté de laisser s'exprimer les recherches architecturales selon les contraintes du terrain. En dépit du clocher, ces bâtiments n'aspirent nullement à la monumentalité, tout au contraire, Notre-Dame de Pentecôte fait le pari, tout en étant en harmonie avec les façades de verre de La Défense, d'être le plus petit édifice de la place, inversant le rapport ancien entre l'église et les bâtiments alentours. Comme l'immense croix dessinée en ombre sur la voile de verre qui s'élève devant l'édifice n'était pas également visible selon les heures du jour et surtout selon l'orientation d'arrivée à la sortie du RER, elle a été doublée, à la demande des

19. Avec une visibilité plus forte que l'ancien « relais Jean XXIII», enfoui en sous-sol d'une tour. Voir http://catholiques-aladefense.cef.fr; http://www. franck-hammoutene.com.

20. Outre les publications signalées en référence, un DVD (film réalisé par G. Tomczak) a été édité par l'association "Art, Culture et Foi, Paris », à l'initiative d'Isabelle Renaud-Chamska, sous le titre «Sept nouvelles églises pour aujourd'hui » (la chapelle de l'Agneau de Dieu, près de la gare de Lyon, y est également présentée). 
fidèles, d'une grande croix métallique plus visible. Dans chacun des cas, le souci d'une présence affirmée, mais sans démesure, signe ce retour d'une identité ecclésiale inscrite dans l'espace de la cité.

\section{LA FORME DE L'ASSEMBLÉE}

Bien que certains aspects innovants aient pu surprendre, ces projets longuement mûris ont fait l'objet d'échanges précis avec les commanditaires. Mgr Lustiger a notamment suivi de près l'aménagement de Notre-Dame-de-l'Arche-d'Alliance, de Notre-Damede-la-Sagesse ou de Saint-François. Pour l'espace intérieur, plus directement au cœur de notre sujet, la conception de l'architecte a toute son importance et traduit également la diversité des formules qui viennent en écho à la réforme conciliaire. L'insistance mise par la constitution sur la liturgie sur la « participation pleine et active ${ }^{21}$ » de l'assemblée (SC 11) a favorisé la réflexion, déjà sensible bien avant le concile, notamment chez les architectes proches du théologien Romano Guardini, sur la notion d'espaces actifs et sur la forme de l'ecclesia ${ }^{22}$. Il s'agit de tendre vers un modèle moins hiérarchique, inspiré de la forme conventuelle de l'assemblée célébrante ${ }^{23}$. Le modèle des églises «tentes » dont on trouve plusieurs exemples en banlieue parisienne ou des églises rondes, telle la cathédrale d'Évry, ne se retrouve pas ici, en partie en raison des contraintes de terrain réduit à l'espace de la rue parisienne. Toutefois, à l'exception de la chapelle Notre-Dame-de-la-Sagesse, qui ne comporte qu'une dizaine de bancs, l'organisation intérieure privilégie une disposition enveloppante, sensible à Notre-Dame de Pentecôte qui place le sanctuaire au premier étage du bâtiment, à l'instar de la chambre haute du cénacle antique, mais également dans les autres édifices qui tirent parti des contraintes de la présence au sol pour élever l'assemblée sur plu-

21. Yves Congar, "L'Ecclesia ou communauté chrétienne sujet intégral de l'action liturgique » dans La Liturgie après Vatican II, Paris, Le Cerf, «Unam Sanctam », 66, 1967, p. 241 et sq.

22. Romano Guardini, Vom Geist der Liturgie, 1918; voir notamment Frédéric Debuyst, L'Entrée en liturgie : introduction à l'œuvre liturgique de Romano Guardini, Paris, Le Cerf, 2008.

23. Isabelle Saint-Martin, "L'évolution de l'espace cultuel» dans Céline Frémaux (dir.), Architecture religieuse au XX $x^{e}$ siècle. Quel patrimoine?, INHA et Presses universitaires de Rennes, 2007, p. 31-39. 
sieurs niveaux avec un plan en carré et des gradins à Notre-Damede-l'Arche-d'Alliance, des tribunes à Saint-François de Molitor, ou un simple balcon à Saint-Luc. L'étagement des participants et la présence de bancs sur les côtés qui encouragent un dispositif en visà-vis contribuent à matérialiser la forme de l'ecclesia. La référence à l'intimité du modèle monastique est d'autant plus appropriée que ces églises s'adressent à des communautés à taille humaine et non au large découpage paroissial de la fin du XIX ${ }^{e}$ siècle, qu'il s'agisse des deux cent trente fidèles pour Saint-Luc, ou de près de quatre cents à Notre-Dame-de-l'Arche-d'Alliance. Toutefois, la différence est nette entre les partis pris des différents édifices. À l'Arche d'alliance, le centre est marqué par une croix formée de dalles de verre qui laissent apercevoir le baptistère situé en dessous, le plan en forme de croix grecque est orienté vers l'abside où se trouvent l'autel et de l'ambon (fig. 2). Un croisillon blanc très largement espacé rappelle la résille extérieure du narthex et distingue l'espace de célébration, de même à Saint-Luc, à La Sagesse ou Notre-Dame-d'Espérance, la disposition est relativement frontale, les bancs faisant face au chœur. Dans l'esprit du concile, l'autel est toujours suffisamment avancé pour qu'on puisse en faire le tour ${ }^{24}$ et célébrer face au peuple, l'ambon a conquis un espace propre, à juste distance de la «table du repas eucharistique » pour donner à ce qui est parfois appelé « table de la parole » la place qui lui revient dans la nouvelle liturgie. Le troisième pôle que constitue le siège de la présidence cherche parfois encore son équilibre, avec des solutions innovantes telles le banc arrondi qui souligne l'espace de gloire, dégagé derrière l'autel, à l'Arche d'alliance. La croix sobre et lumineuse qui rythme cet ovale laisse deviner une forme de visage inspiré des expressions minimalistes de Jawlensky. De la même manière, à Notre-Dame-d'Espérance, l'architecte a eu le souci de ménager un espace courbe entièrement blanc avec une lumière zénithale derrière l'autel (fig. 1). Ce choix d'un prolongement du regard et d'une ouverture au-delà du sanctuaire, que l'on peut qualifier d' " espace de gloire ${ }^{25}$ » tend à répondre à la critique d'une focalisation excessive des regards vers le célébrant, sur le modèle de la salle d'école, avec un risque d'autocélébration de l'assemblée pour elle-même et non vers ce pour quoi elle est réunie.

24. Instruction Inter Oecumenici du 26 septembre 1964 (chap. V, n ${ }^{\circ}$ 1).

25. Frédéric Debuyst, Le Génie chrétien du lieu, Paris, Le Cerf, 1997. 
Une tout autre solution est proposée lorsque les trois pôles, ambon, autel, siège de la présidence, se trouvent placés sur le même axe entourés de part et d'autre par l'assemblée. Ce choix mis en œuvre à Notre-Dame-de-Pentecôte dans le contexte d'une petite communauté non paroissiale permet une disposition en vis-à-vis orientée vers le lieu de la célébration (fig. 6). Jean-Marie Duthilleul, après avoir également testé cette forme ovoïde ${ }^{26}$, propose, pour Saint-François de Molitor, un plan en ellipse dans lequel le baptistère, qui signe le seuil, se trouve exactement dans l'axe de l'ambon et de l'autel, donnant à l'assemblée, répartie de part et d'autre, la forme d'une mandorle (fig. 5).

\section{CONSTRUIRE DES ESPACES SIGNIFIANTS : LE RAPPORT INTÉRIEUR/EXTÉRIEUR}

Visibilité extérieure affirmée et souci d'un aménagement intérieur pensé selon une perspective liturgique précise forment les deux pôles d'une réflexion sur le rapport entre intérieur et extérieur, sur ce qui distingue et sépare le bâtiment église du reste de la rue, mais aussi sur ce qui l'ouvre à l'extérieur et signe une présence au monde qui ne soit pas repli sur soi. Cette double articulation se traduit de façon manifeste dans la conception de ces édifices. Le soin attaché d'une part aux façades et d'autre part à l'aménagement d'espaces de transition en témoigne. La résille métallique qui rappelle l'esprit d'un narthex autour de l'Arche d'alliance en est un exemple frappant, mais la notion de seuil et de lieu d'accueil apparaît selon les possibilités dans chacun des cas, tandis que, dans un mouvement symétrique, les revêtements extérieurs sont étudiés de manière à laisser entrer un jeu de lumière ou tout au moins à manifester une forme de porosité. Ainsi, le marbre translucide de la façade sur la rue à Saint-François de Molitor trouve un écho dans la verrière du mur $\mathrm{du}$ fond au grain travaillé de façon à masquer en partie la vue sur les bâtiments environnants tout en laissant deviner leur présence, et en s'affinant en partie haute pour faire entrer le maximum de lumière naturelle (fig. 5 et 11). De la même manière, la voile de béton et de

26. En participant aux expériences pour l'aménagement du chœur de SaintIgnace, voir Claire Génin, Chroniques d'art sacré, n 83, automne 2005, p. 19-20. 
couches de verre qui protège la maison d'église de La Défense du bruit incessant de l'esplanade, ne descend pas jusqu'au sol afin de ménager un espace interstice à la fois lumineux et ouvert sur la vie bouillonnante de ce quartier. À la Sagesse, le grand mur de brique rouge sur la façade ouest laisse un jour en bas qui évite une séparation trop marquée avec le milieu environnant. Selon l'architecte P.-L. Faloci : « un mur suspendu plonge ses piliers dans un bassin qui recueille l'eau du ciel. En se réfléchissant sur l'eau la lumière tamisée fera vibrer l'église ${ }^{27} \gg$.

Le cas le plus frappant est celui de la façade de l'entrée principale de Notre-Dame-d'Espérance, composée de plusieurs couches de verre calligraphié par Franck Lalou dans une écriture en boustrophédon. Les versets des évangiles inscrits sur ce mur extérieur se lisent une ligne sur deux, tandis que l'on devine sur l'autre ligne que l'écriture se poursuit en miroir, lisible de l'intérieur. Ce qui se donne à voir de l'extérieur invite à la découverte, mais pour prolonger la lecture, il faut franchir le seuil et pénétrer dans l'église.

Cette recherche d'ouverture, comme l'emploi du béton poli et du verre, ne sont certes spécifiques ni de ces exemples ni de l'architecture religieuse; la réflexion sur les édifices publics et les bâtiments administratifs est similaire. Il reste que l'équilibre entre la volonté d'affirmer une présence et le souhait de manifester l'ouverture au monde en signifiant une interpénétration des regards s'inscrit dans les aspirations actuelles de l'église. Il s'agit de rendre sensible la présence des préoccupations chrétiennes dans l'univers urbain quotidien, mais aussi de recevoir au sein du sanctuaire les échos du monde extérieur. L'ouverture vient en corollaire d'un souci de distinction qui ne s'exprime pas seulement par les espaces d'accueil qui visent à matérialiser la notion de passage et donc affirmer une différence entre l'intérieur ecclésial et le monde environnant, mais aussi par un ensemble de détails signifiants.

MAtériauX ET MOBILIER LITURGIQUe : « LA NOBLE BEAUTÉ »

Le choix de matériaux en est un signe aisément perceptible. À Saint-François de Molitor dominent le marbre de l'autel et du 
baptistère, le bois de chêne des bancs de l'assemblée, d'acajou pour ceux de la présidence, de sycomore au plafond, la pierre brute des murs (fig. 5). Brique et bois encore à la Sagesse dont l'espace dépouillé s'inspire de Le Corbusier, marbre de Carrare à NotreDame-d'Espérance pour le chœur, acajou pour le plafond, le choix de matériaux nobles ou considérés comme authentiques contribuent à caractériser l'espace afin de le distinguer des univers environnants. Il correspond aussi, en principe, pour le mobilier liturgique aux prescriptions rappelées dans la Présentation générale du missel romain: «Selon une coutume et un symbolisme traditionnels dans 1'Église, la table d'un autel fixe sera en pierre et même en pierre naturelle. Cependant on pourra aussi employer, au jugement de la Conférence des évêques, un autre matériau digne, solide et bien travaillé $^{28} .$. ». Si le marbre est très présent, tel le marbre de Tassos à l'Arche d'alliance ${ }^{29}$, le recours à des matériaux contemporains n'est pas exclu pour des œuvres très stylisées dont la dimension symbolique est mise en valeur. L'aluminium poli de l'autel, repris aussi pour l'ambon, le tabernacle et le baptistère ${ }^{30}$, réfléchit intensément la lumière à Saint-Luc (fig. 3). En effet, confronté à une surface restreinte, l'architecte a magnifié l'espace du chœur par l'élévation d'une verrière de 13 mètres. La lumière zénithale tombe sur une colonne de vitrail jaune-vert qui colore l'espace. Un pan découpé dans l'autel oriente le regard vers le grand crucifix (école allemande du XVII $\left.{ }^{\mathrm{e}}\right)^{31}$ placé à côté. Le cube d'acier massif de l'autel de NotreDame de Pentecôte est travaillé de larges entailles qui suggèrent les lames de feu descendant sur les apôtres (Pierre Sabatier), tandis que l'ambon en métal patiné est ciselé pour évoquer le feu du buisson ardent dont jaillit la parole divine (fig. 6).

La plupart de ces autels ont une forme cubique et ramassée suggérant une présence sans monumentalité dans l'esprit prôné en France par le mouvement liturgique ${ }^{32}$. Si les formats varient avec plus ou moins de verticalité, le seul à jouer de l'originalité dans un

28. L'Art de célébrer la messe. Présentation générale du missel romain, Paris, Desclée, 2008, chap. V. n 301.

29. Anne-Marie de La Soudière, «L'autel, lieu de l'altérité », Chroniques d'art sacré, $\mathrm{n}^{\circ}$ 78, 2004, p. 23.

30. Design du cabinet MATO (Bernard Malaquis).

31. Placé provisoirement dans le chœur, offert par un antiquaire.

32. Voir Frédéric Debuyst, « La problématique de l'autel », brochure extraite des Chroniques d'art sacré, 1985. 
domaine où la noble simplicité semble de bon aloi, est l'autel conçu par le sculpteur François Cante-Pacos à Notre-Dame-d'Espérance (fig. 1). Une forme ovale scindée en deux évoque la terre divisée que la table eucharistique réunit, suggérant également la rencontre des mondes célestes et terrestres. Les autres éléments du mobilier liturgique sont confiés au cabinet de design Xylos. La simplicité des formes géométriques accompagne les bois précieux, ainsi le tabernacle en acacia doré joue sur le symbole de l'arche d'Alliance, alors que depuis les années soixante autels et ambons avaient pu être le support de décors iconographiques liés au repas eucharistique ou à la prédication apostolique avec des symboles stylisés revenant souvent à l'iconographie primitive (poisson, vigne...), tendance encore très présente dans certains décors (ambon avec les symboles des évangélistes à Notre-Dame-d'Espérance).

\section{QUELLE PLACE POUR LES INTERVENTIONS D'ARTISTES?}

Le sens du dépouillement, la crainte d'excès dévotionnels et l'intérêt pour la dimension spirituelle de l'abstraction ont contribué, depuis les années soixante, à minorer la place de l'iconographie dans les églises. Mais elle n'est pas ici absente, réclamée notamment par les fidèles. Ainsi, à la demande des paroissiens, ont été posés à l'Arche d'Alliance des vitraux narratifs, confiés à un artiste du pop art, Martial Raysse ${ }^{33}$. Deux grandes baies $\left(36 \mathrm{~m}^{2}\right)$ «David dansant devant l'arche » et la «Visitation » jouent sur le vocable de l'église et transforment l'atmosphère par un jeu de couleurs acidulées dessinant les formes en larges aplats. Cependant l'essentiel de l'apport des artistes reste du domaine de la sculpture avec la question particulière du crucifix et de la Vierge. Dans la majorité des cas (à l'exception de Saint-Luc cité plus haut), la croix, nécessairement présente à côté de l'autel, est totalement dépouillée. Croix nue, elle se veut signe de gloire autant qu'instrument du supplice, rappel de ce que celui qui y fut attaché est celui qui relie les quatre orients, selon la réflexion patristique. Qu'il s'agisse de la grande croix de lumière qui dessine l'esquisse d'un visage à l'Arche d'Alliance ou

33. La commission diocésaine d'art sacré de Paris a organisé le concours en demandant que le sujet reprenne le thème de l'Arche d'alliance. La réalisation a été confiée à l'atelier Jean-Dominique Fleury. 
de la croix grecque recouverte de particules d'or, monumentale et glorieuse, mais comme en suspension au-dessus du chœur à NotreDame-de-la-Sagesse (fig. 4), ou tout au contraire de la mince et haute croix d'acier doré à l'or fin, à Saint-François, ou encore du Christ confondu avec la forme de la croix, émergeant tel le surgeon de David (Is 53), conçu par Pierre de Graw à La Défense (fig. 6), ces croix refusent tout pathos et préfèrent le silence, l'absence de la figure christique pour signifier l'espérance de la résurrection. Cette esthétique du dépouillement trouve une forme limite avec la croix du sculpteur Nicolas Alquin ${ }^{34}$ à Notre-Dame-d'Espérance (fig. 1). Seule la poutre verticale, un bois du XVIII ${ }^{\mathrm{e}}$ siècle longuement patiné, est fichée derrière l'autel, à l'instar de la crux antique. La barre horizontale, celle que portait le condamné, fait ici défaut. Elle n'est que virtuellement présente par trois plots dorés, soit deux carrés dans les montants architecturaux de l'église à gauche et à droite de la poutre, rejoignant, en une ligne imaginaire, le carré central dessiné sur la poutre. La travée manquante est à réinventer toujours dans le regard du visiteur qui, dès le franchissement du seuil, est invité à se déterminer face à cette croix virtuelle, à investir de son regard et de ses attentes l'univers visuel dans lequel il pénètre, à saisir sa croix pour suivre le Christ.

Outre la croix, toutes ces églises ont également un crucifix, qui est, par contraste, le plus souvent de haute taille, en bois et de facture ancienne $\left(\mathrm{XV}^{\mathrm{e}} \text { au } \mathrm{XVII}^{\mathrm{e}}\right)^{35}$, placé en général dans un espace en retrait, un dégagement latéral avec le tabernacle à Saint-François de Molitor, ou un angle peu visible de l'assemblée, afin de permettre une prière personnelle et intime devant la figure christique. Une Vierge est également toujours présente, même si ce n'est qu'un visage et le fiat des mains jointes selon la proposition originale du sculpteur Étienne à Notre-Dame-de-Pentecôte. À Saint-Luc, l'espace de prière ne proposait qu'une icône de la Vierge (selon une tendance affirmée depuis plusieurs décennies ${ }^{36}$, ces icônes, que l'on trouve aussi à La Sagesse et Notre-Dame-de-Pentecôte, apparaissent comme un substitut à l'ascèse iconographique de l'espace);

34. Paul-Louis Rinuy, "Une croix pour l'espérance», Chroniques d'art sacré, 2003, n 75 , p. 30.

35. Ou de style rappelant d'anciens crucifix.

36. Voir François Boespflug, «Le retour de l'icône», dans Jean-Michel Spieser (éd.), Présence de Byzance, Paris, Gollion, 2007. 
un buste de Marie tenant tendrement son fils, haut-relief en bronze, offert par un fidèle ${ }^{37}$, a été rajouté et manifeste toute l'importance qu'une communauté attache à ces objets, pivots de la dévotion individuelle canalisant regard et prière et assurant l'articulation du recueillement privé et de la prière communautaire. Selon les lieux apparaissent d'autres sculptures, tel un saint François (Alain Courtaigne) à Saint-François de Molitor, un songe inspiré du Cantique des Cantiques à l'Arche-d'Alliance, ou encore la statue de Notre-Dame-d'Espérance et l'ancien chemin de croix, réalisés dans les années trente par Lucienne Heuvelmans, pour l'église de la rue de la Roquette, où ils rejoignent les vitraux sur le thème du désert de Jean-Baptiste Ambroselli, mais toujours selon un principe général d'économie. Ainsi, à Notre-Dame-de-Pentecôte, l'architecte restait réservé à l'idée d'introduire un vitrail coloré derrière l'autel. C'est finalement une forme stylisée, de grandes dynamiques évoquant le souffle de l'Esprit, sculptées par Jacques Loire (fig. 6), qui se développent devant la baie, formant une sorte de vitrail métallique qui ne détourne pas l'attention de l'autel et de la croix en matériaux accordés et laisse se déployer, en écho latéral, la présence colorée des grandes tapisseries du Frère Yves. À Notre-Dame-de-la-Sagesse, si l'on trouve bien sur le parcours latéral un triptyque de La Transfiguration $^{38}$ de style abstrait, c'est un grand panneau doré qui prend place derrière l'autel (fig. 4) et réfléchit la lumière sans déparer l'atmosphère cistercienne du lieu, où l'austérité de la brique fait référence à Le Corbusier, alors que les deux très belles statues anciennes qui accompagnent la prière : la Vierge bourguignonne $d u \mathrm{Xv}^{\mathrm{e}}$ dans un renfoncement à gauche de l'autel ou le Christ portugais du $\mathrm{XvI}^{\mathrm{e}}$ dans l'angle réservé à la réconciliation sont invisibles au premier regard lorsqu'on pénètre dans l'église.

Les artistes sont donc présents, mais avec des œuvres choisies en nombre limité afin de laisser à chacune d'elles un espace de résonance, en évitant tout effet d'accumulation dans des espaces restreints. Le respect de la composition d'ensemble conçue par l'architecte domine. S'il est juste de parler d'une esthétique de la sobriété, il faut comparer ces ambiances soigneusement travaillées, où marbre et bois précieux se répondent, aux installations provi-

37. Créé par Laurence Leplay, juin 2002.

38. De Jean-Rodolphe Loth. 
soires des années soixante-dix pour mesurer le chemin parcouru dans l'appréciation de la notion de dépouillement. Pourtant c'est bien à ce terme que se réfère J.-M. Duthilleul, opposant, selon l'esprit des origines du mouvement liturgique, « la profusion des signes » qui règne dans la capitale à la recherche d'essentiel qui doit animer la conception d'une église :

Pour être fort, aujourd'hui, il faut sans doute se taire! En pénétrant dans une église, on entre dans quelque chose qui a trait à l'essentiel. Et l'essentiel n'est pas bavard! Dieu n'est pas dans la tempête, dans l'agitation, il est dans le murmure. Une composition très dépouillée permet sans doute de mieux l'écouter ${ }^{39}$.

L'idéal esthétique de ces réalisations se situe bien davantage dans la composition harmonieuse d'un ensemble que dans la présence, ou pire la juxtaposition, d'œuvres si belles soient-elles, qui mettraient surtout en valeur l'artiste concepteur. Vient alors à l'esprit l'invitation de Jean-Yves Hameline à une esthétique de l'aptum ${ }^{40}$ (à la manière des stoïciens) soit du juste rapport, de la congruence en toute chose, plutôt qu'une esthétique du pulchrum (de la beauté en soi et pour elle-même). C'est au contraire dans ce juste équilibre que se manifeste la quête de beauté de ce dispositif ecclésial.

\section{Du VISIBLE AU LISIBLE : AFFIRMER UNE LECTURE SYMBOLIQUE}

Évoquer tour à tour la forme de l'assemblée, le jeu entre intérieur et extérieur, et les principaux éléments du décor, afin d'examiner la construction de ces espaces signifiants, clairement distingués de l'architecture environnante, a conduit à laisser de côté certains aspects de la signification symbolique attachée à ces lieux. Y revenir à la fin de ce parcours invite à reprendre les détails de l'aménagement pour les intégrer dans une lecture d'ensemble qui n'est peut-être pas immédiatement perceptible par le fidèle ou le visiteur, mais qui lui est néanmoins clairement proposée, notamment dans les petits dépliants disponibles à l'accueil, les visites guidées, ou les commentaires parus dans la presse catholique. Ainsi pour l'église de la rue d'Alleray, c'est au cours du projet, devant le volume

39. $P N D, \mathrm{n}^{\circ} 1083,17$ mars 2005 , « Une nouvelle église à Paris ». p. 86

40. Jean-Yves Hameline, Une poétique du rituel, Paris, Le Cerf, 1997, 
cubique, revêtu de panneaux en fibre de bois et résine, proposé finalement par les architectes, que le vocable, initialement suggéré, de Notre-Dame-de-la-Résurrection s'est transformé pour reprendre un des qualificatifs des Litanies de la Vierge. À l'arche réceptacle de la première alliance fait écho la figure mariale, réceptacle du verbe de Dieu. La forme de coffret précieux pris par cette église montée sur pilotis ne renvoie à aucun type classique. Mais ces douze piliers sont reliés aux douze apôtres et aux douze tribus d'Israël qui supportent ce cube, figure de la Cité sainte (Ap., 21, 9-21). Cette église ainsi conçue « renvoie à une autre réalité qu'elle-même ${ }^{41}$ ", ce qui est la définition du symbole (« deux réalités distinctes qui renvoient l'une à l'autre »). Le baptistère placé au centre des douze piliers, comme en soubassement de l'édifice, évoque la place du baptême comme fondement de toute vie chrétienne, signe de l'entrée dans le peuple de Dieu. La place de l'initiation baptismale se retrouve dès l'accueil à Saint-François de Molitor, dans l'axe de l'autel, ou encore au pied de l'escalier à Notre-Dame-de-Pentecôte. En principe, devaient sourdre du mur quatre filets d'eau ${ }^{42}$, recueillis dans une cuve baptismale puis sur la dalle noire portant un verset d'Isaïe (28, 16-17), montrant le sens du baptême comme pierre angulaire de l'entrée dans la foi.

Cette symbolique imprime également sa marque à tout le côté gauche de la chapelle Notre-Dame-de-la-Sagesse. Le long d'une cloison de verre, l'espace est divisé en quatre paliers ou «chapelles », faisant se succéder le baptistère, la table des saintes huiles, le cierge pascal de la résurrection, puis le vêtement blanc des nouveaux baptisés évoqué par une haute feuille de marbre. Ce chemin baptismal, bordé par un mince bassin d'eau à gauche, mène à la statue de la Vierge qui semble ainsi guider le baptisé vers le tabernacle et l'autel. Symétrique au baptistère, sur la droite, la chapelle de la réconciliation est située sous le clocher. Après avoir médité devant le Christ du $\mathrm{Xv}^{\mathrm{e}}$ siècle, le fidèle trouve dans sept fentes de lumière du mur droit, le long des bancs, sept paroles de la Passion ${ }^{43}$. Gravées verticalement, elles forment comme un chemin de croix, un chemin du pardon qui conduit à l'autel.

41. $P N D, \mathrm{n}^{\circ} 738,18$ juin 1998, «La Nouvelle Arche d'Alliance», propos du P. E. Schwab.

42. Dans la pratique cet écoulement permanent n'est pas toujours possible.

43. Sept dernières paroles du Christ prises dans les quatre évangiles. 
La place des inscriptions tracées sur les murs mérite d'être soulignée. Elle révèle l'attention particulière portée à la Parole dans la réforme conciliaire, mais aussi l'iconicité de l'écriture, signe doté de sa propre esthétique comme le montrent nombre de variations sur la calligraphie dans l'art contemporain, associant les références à l'art médiéval ou à la poétique visuelle des calligrammes. Les versets prennent toute leur valeur ornementale et proclament la destination de l'édifice sur la façade de Notre-Dame-d'Espérance (calligraphiée par Franck $\mathrm{Lalou}^{44}$ ), ou encore à travers la répétition psalmodique du « Je vous salue Marie » sérigraphié sur la face principale du cube de l'Arche-d'Alliance, comme dans les citations de l'évangile de Luc et Actes des apôtres qui s'impriment sur les deux vantaux de la porte de Saint-Luc.

Donner à voir et à comprendre, à la manière des tympans sculptés des cathédrales, réaffirme la signification d'une architecture qui veut ses murs porteurs de la spiritualité qui anime l'intérieur du sanctuaire. L'effet est d'autant plus manifeste à Saint-Luc, où l'église étroite, ne se distingue guère en façade que par son haut clocher, mais le dépliant d'accueil prend soin de rappeler que « la façade en verre est divisée en quatre colonnes, signifiant les quatre évangiles [...], Saint Luc est le troisième aussi la porte d'entrée est-elle dans la troisième colonne. »

\section{ForMER LES CADRES DE L'EXPÉRIENCE RELIGIEUSE}

Cette volonté de donner sens au moindre détail dans l'aménagement de l'espace, de conférer à chaque élément matériel une signification symbolique, vise à accompagner le fidèle dans sa découverte des lieux. Ainsi conçue, la traversée de l'église s'apparente à un parcours initiatique qui offre une lecture des mystères chrétiens. Il s'agit d'une certaine manière de former les cadres de l'expérience sensorielle afin de guider l'expérience religieuse. À Notre-Damede-la-Sagesse, l'allée centrale recouverte de granit poli suit une pente inclinée sur laquelle la lumière se reflète lorsqu'on avance dans la chapelle, «symbole d'une descente spirituelle à l'intérieur

44. Versets des évangiles sur la façade de verre, rue de la Roquette, textes de l'Ancien Testament sur le mur de la rue du Commandant-Lamy. Voir Chroniques d'art sacré, 2001, n 68 , p. 19-20. 
de $\operatorname{soi}^{45} »$. Selon les mots d'un paroissien: "On se sent comme aspiré vers l'aute ${ }^{46}$. » Inciter à ressentir davantage, au travers de l'expérience corporelle, la perception de l'espace cultuel et le sens du rite rejoint à bien des égards les souhaits de Romano Guardini qui déplorait en son temps l'appauvrissement de la dimension concrète du spirituel. Dans Les Signes sacrés, il insistait certes davantage sur la montée (les degrés ${ }^{47}$ ) que sur la descente, mais surtout son mode d'interprétation était d'abord anthropologique et soucieux du sens du geste, alors que l'aspect pédagogique et biblique est plus manifeste dans les commentaires de ces nouvelles églises. Ainsi, lorsqu'à Saint-François, l'architecte C. Callies souligne que «l'église est de plus en plus lumineuse au fur et à mesure que l'on s'avance vers la croix », Mgr Lustiger compare le plan en ellipse légèrement incurvé à la barque de Pierre dont la croix serait la proue, le baptistère la poupe et l'autel, la quille ${ }^{48}$.

Manifester une continuité entre le signe visible et son interprétation répond à cette volonté de proposer une réalité qui renvoie à autre chose qu'elle-même. Or selon la pensée de Mgr Lustiger, qui ne fut certes pas le seul concepteur de ces lieux, mais le principal commanditaire, il y a là un point de rencontre entre l'expérience esthétique et la pratique sacramentelle. Pour lui, « la beauté ne prétend pas capter l'attention de celui qui regarde [...], mais renvoyer à ce que l'œil ne peut pas voir ». Elle met en marche vers l'invisible : «Ce qui est signifié transfigure ce que l'artiste a fait. » Pour l'expliquer, il fait un parallèle heuristique : «De même dans l'acte sacramentel de la messe, ce qui est visible est fragile et fugace [...] Mais ce qui est signifié est infiniment plus grand et plus fort $[\ldots]$ Le sacrement est en quelque sorte une œuvre d'art réduite à sa plus extrême sobriété d'expression. Un acte esthétique du Christ où les hommes reçoivent en partage la suprême beauté de la Vie ${ }^{49}$. »

Dans cette perspective, le sommet de l'art est la liturgie ellemême, mais l'appel à l'art dans l'aménagement du sanctuaire peut contribuer à y conduire ou tout au moins engager la perception sen-

45. Dépliant de présentation de l'église.

46. Entretien avec un visiteur, février 2009.

47. Romano Guardini, Les Signes sacrés, trad. G. Hocquard, université de Metz, 1980, p. 27-28.

48. $P N D, \mathrm{n}^{\circ} 1083$, art. cit.

49. $P N D, \mathrm{n}^{\circ} 1030,19$ février 2004, p. 13. 
sorielle et religieuse de l'édifice. Les œuvres d'art y concourent non pas au sens où elles combleraient des vides dans l'espace, mais au contraire parce que l'art détermine un sens, oriente le regard et « le conduit sans le distraire vers les éléments vitaux de l'organisme liturgique, l'ambon, et l'autel ${ }^{50} \gg$. Il ne s'agit pas d'un décor, mais d'un élément constitutif de l'expérience esthétique propice à l'expérience religieuse.

Faut-il redouter un raffinement excessif des signes qui viendrait outrer l'ambition de ces édifices, à l'orée du XXI ${ }^{\mathrm{e}}$ siècle, de se démarquer de l'église fonctionnelle des années soixante-dix, apte à se satisfaire d'une salle polyvalente? Sans doute y a-t-il des voix pour souhaiter qu'on démonte l' « échafaudage » (la résille qui forme une sorte de narthex) autour de Notre-Dame-de-l'Arche-d'Alliance, que l'on masque la verrière translucide qui laisse deviner les immeubles au fond de Saint-François de Molitor, qu'on rééquilibre la place de l'assemblée par rapport à l'autel à Notre-Dame-de-la-Sagesse, ou encore que la croix trouve une forme pleine à Notre-Damed'Espérance..., pour ne citer que les exemples les plus frappants. Il reste que ces nouvelles églises ont su attirer des communautés actives autour d'elles. Elles l'ont fait en jouant tout à la fois d'une visibilité retrouvée et d'une articulation entre affirmation extérieure et ouverture au monde, manifestant le souci de l'assemblée participante, mais ménageant un espace de recueillement pour la prière personnelle, privilégiant des formes résolument contemporaines, mais associées à la noblesse des matériaux et à la présence soigneusement préservée d'œuvres anciennes traduisant un enracinement au sein de la modernité. La façon d'inscrire chaque élément de l'espace dans une mode d'interprétation symbolique, à la manière des écritures qui sur certaines façades ancrent ces bâtiments dans la lecture biblique, témoigne d'un souci permanent de passage du visible au lisible, du matériel au spirituel. Évoquer la lecture spirituelle de l'édifice ecclésial fait surgir à l'esprit l'ancienne composition du Rationale divinorum de Durand de Mende, si chère encore aux auteurs du XIX ${ }^{\mathrm{e}}$ siècle, mais bien aux antipodes de la réforme conciliaire. D'une autre manière, qui certes n'est ni isolée ni exceptionnelle, mais se dégage clairement de ce corpus, ces édifices, par-

50. François Cassingena-Trevedy, «Éloge de la distance », La Maison-Dieu, 2003/1, p. 43-73. 
faitement ancrés dans leur temps et soucieux de l'esprit de la liturgie prôné par Vatican II, sont également partie prenante d'une volonté de mettre en valeur la spécificité du lieu. Valoriser une forme de sacralité de l'espace ouvre à une dimension pédagogique et pastorale de l'accès à une lecture spirituelle du cadre architectural. Mettre en valeur la dimension scénique de la liturgie et l'importance de la beauté dans l'harmonie générale de l'action rituelle donne à la composition de chacun des éléments inscrits dans une conception d'ensemble, une valeur anagogique. À cet égard, les petits dépliants et les commentaires qui accompagnent ces lieux, participent d'une éducation à la liturgie. Si chaque détail est signifiant et pensé pour distinguer l'église des espaces environnants, si la destination spécifique de l'édifice s'affirme dans sa matérialité, alors déchiffrer le sens des formes prend une valeur mystagogique, qui entraîne l'œil et l'esprit à passer du visible au lisible et par là, à une quête de l'invisible. Celle-ci s'exprime aussi dans l'harmonie de ces lieux intimes, propices à la sérénité, inspirés par le modèle de la communauté monastique, privilégiant les petites assemblées participatives, caractéristiques de la perception actuelle du «petit troupeau »des chrétiens engagés, attachés à rendre sensible la valeur cultuelle des édifices et des œuvres.

ism@ephe.sorbonne.fr 


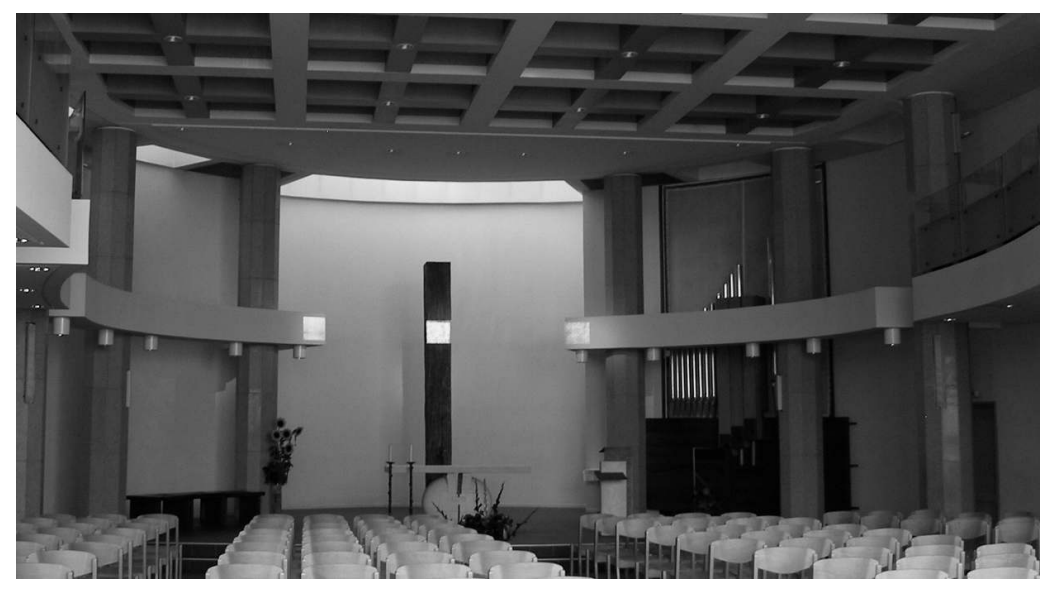

Figure 1. Notre-Dame-d'Espérance, vue intérieure. (47 rue de la Roquette, Paris $11^{\mathrm{e}}$ )

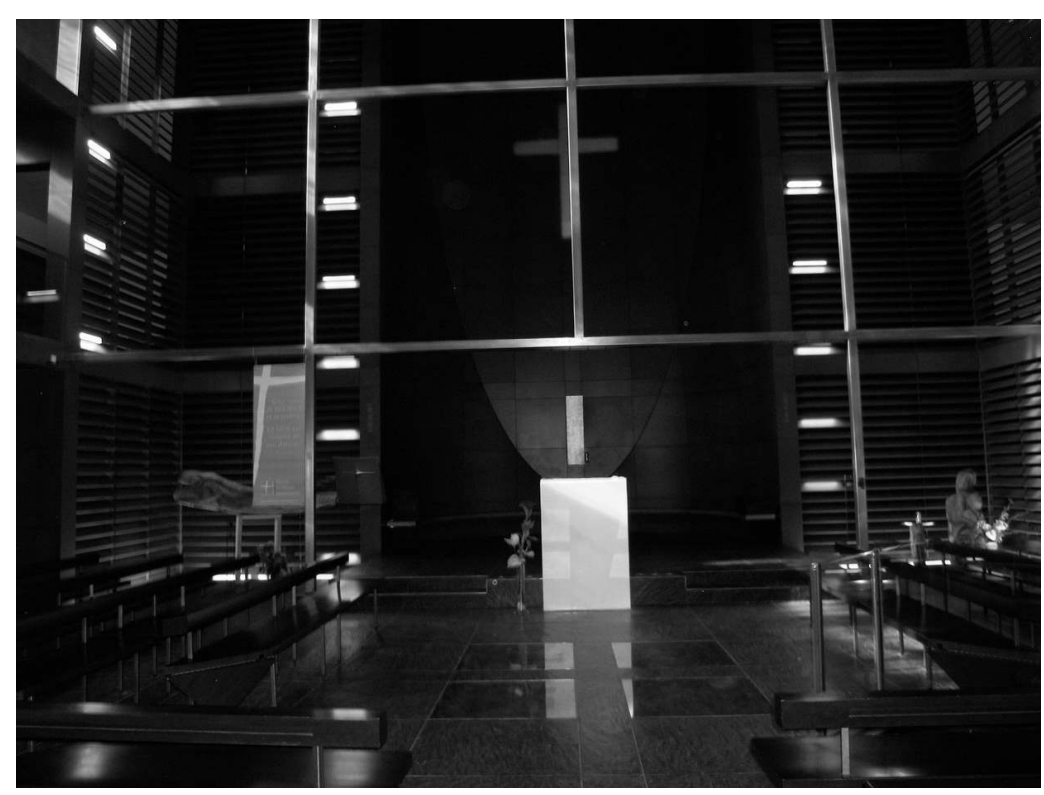

Figure 2. Notre-Dame-de-l'Arche-d'Alliance, vue intérieure. $\left(81\right.$, rue d'Alleray, Paris $\left.15^{\mathrm{e}}\right)$ 


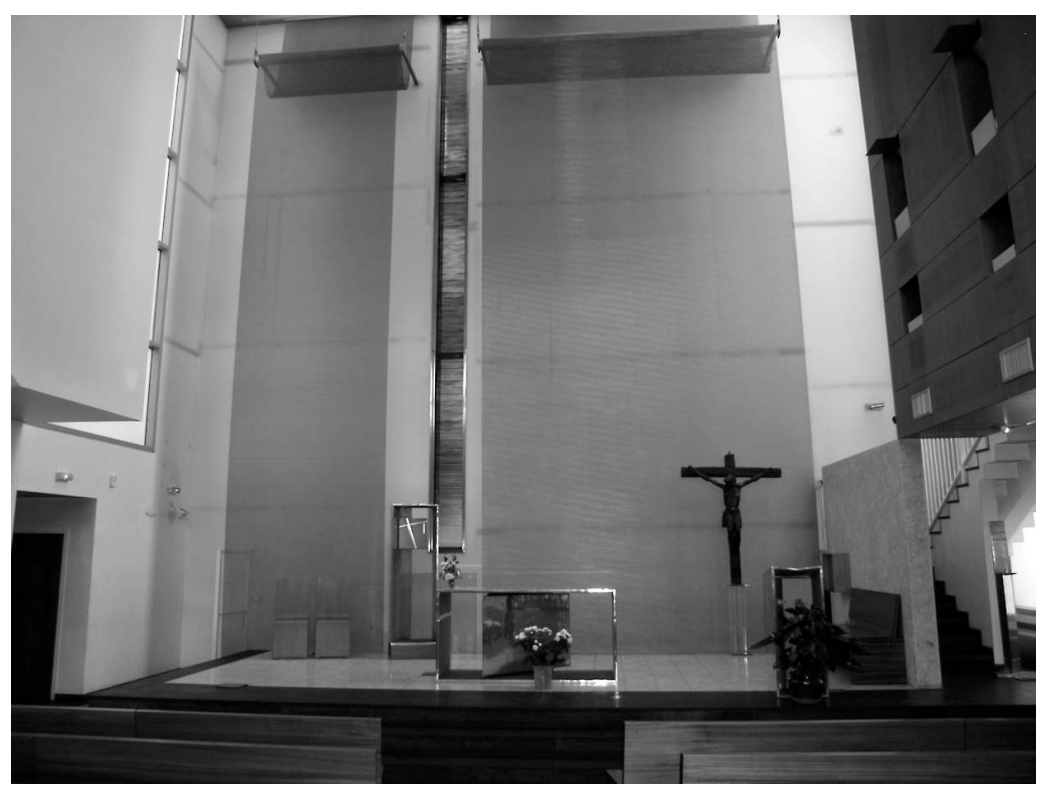

Figure 3. Saint-Luc, vue intérieure.

$\left(80\right.$, rue de l'Ourcq, Paris $\left.19^{\mathrm{e}}\right)$

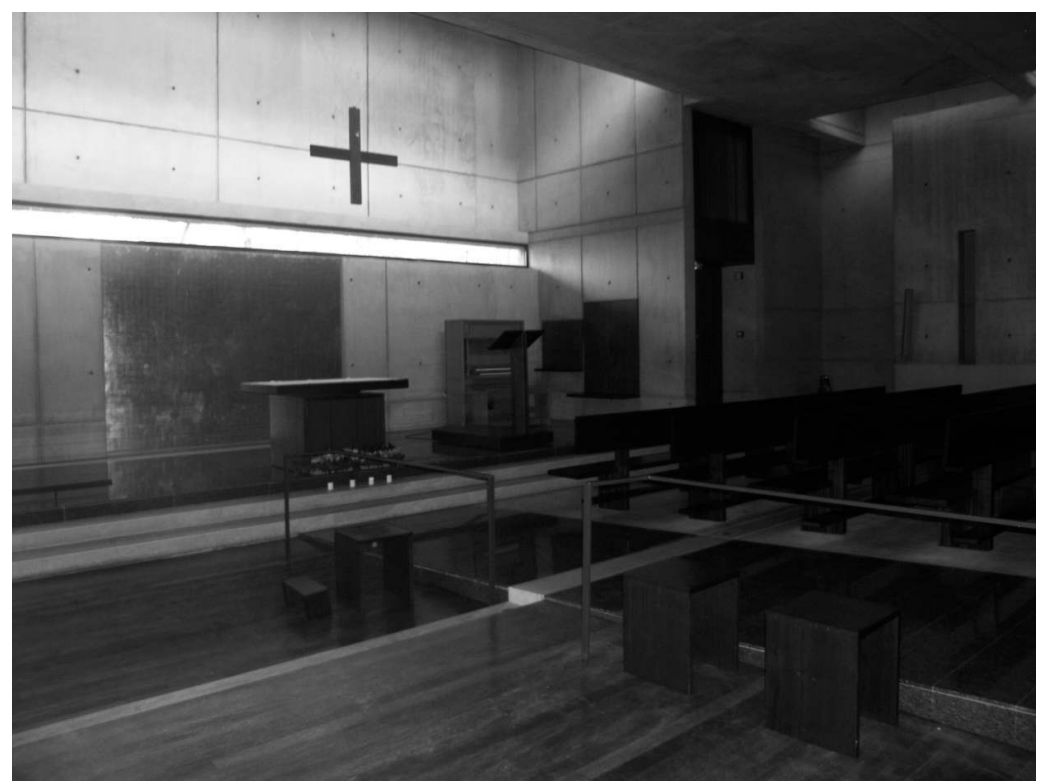

Figure 4. Notre-Dame-de-la-Sagesse, vue intérieure. (13 bis, rue Abel-Gance, Paris $13^{\mathrm{e}}$ ) 


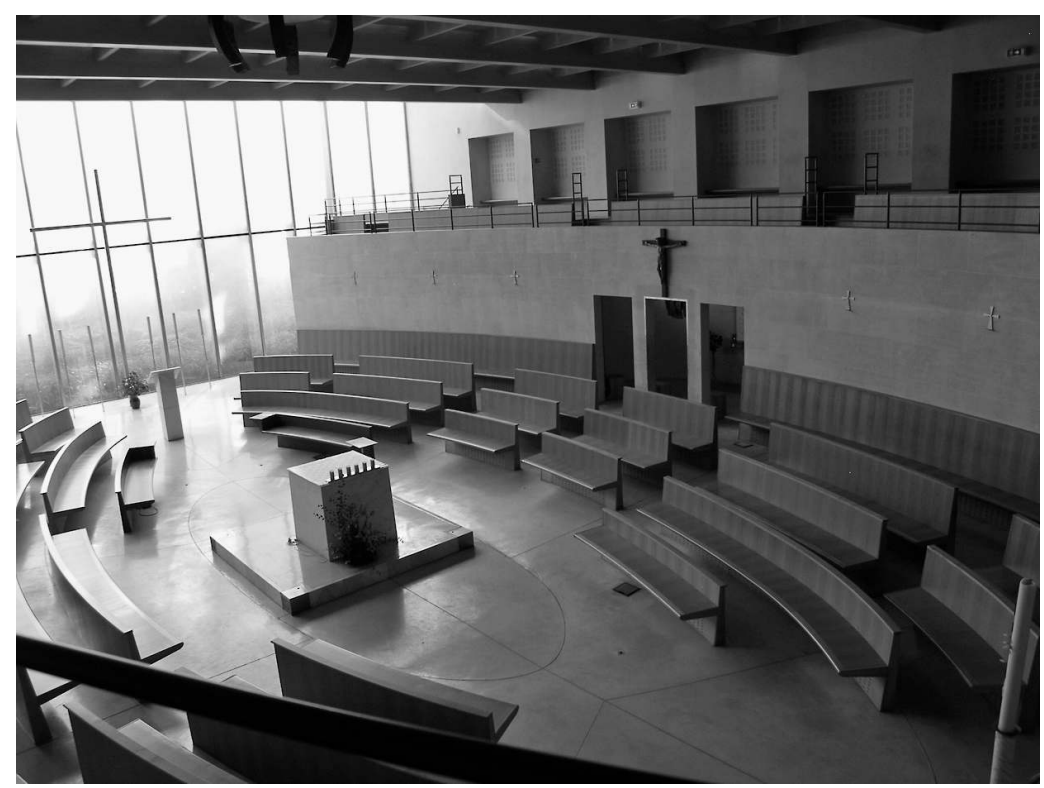

Figure 5. Saint-François de Molitor, vue intérieure. (44, rue Molitor, Paris 16 ${ }^{\mathrm{e}}$ )

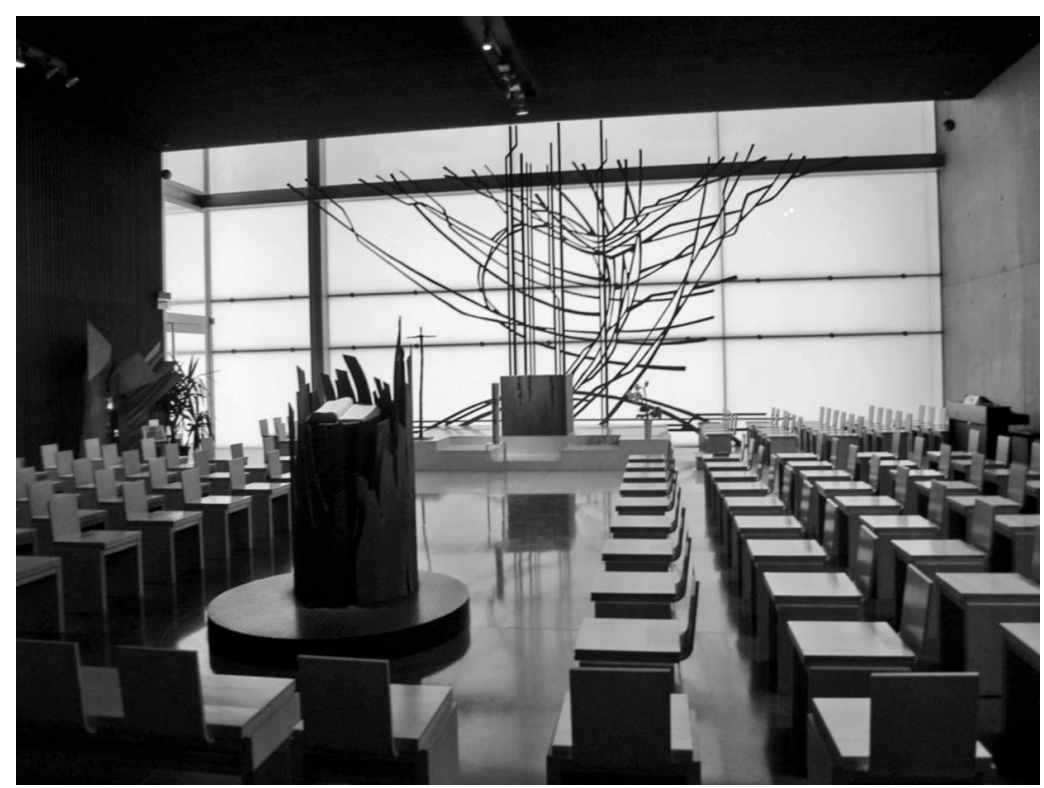

Figure 6. Notre-Dame-de-Pentecôte, vue intérieure. (esplanade de La Défense) 


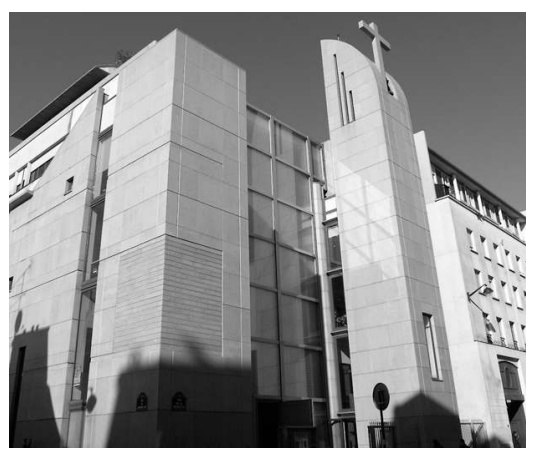

Figure 7.

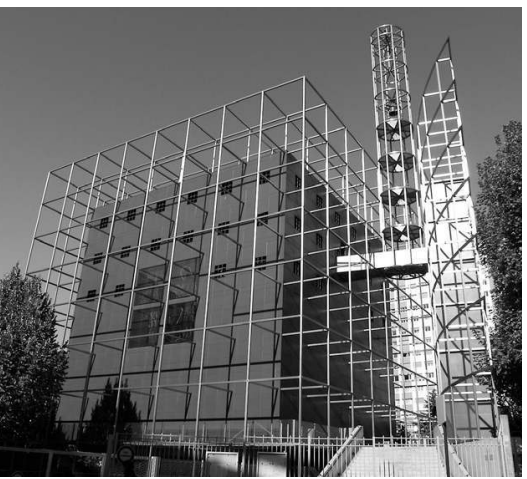

Figure 8 .

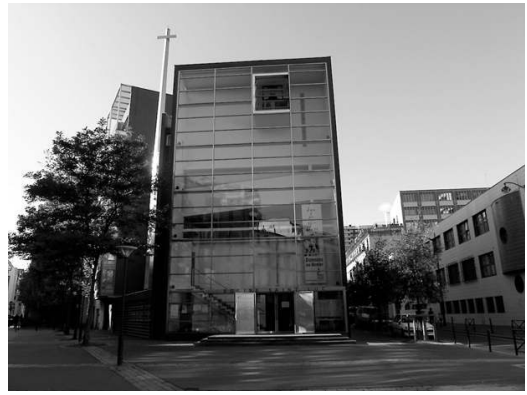

Figure 9.

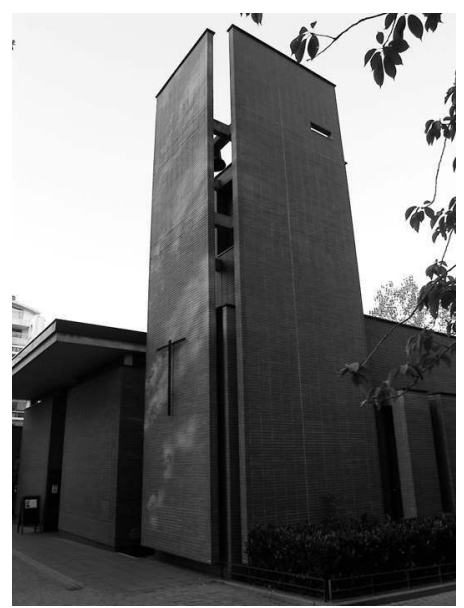

Figure 10.

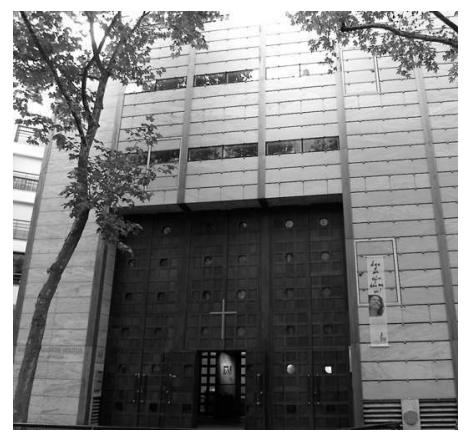

Figure 11.

Vue extérieures

7. Notre-Dame-d'Espérance.

8. Notre-Dame-de-l'Arched'Alliance.

9. Saint-Luc.

10. Notre-Dame-de-la-Sagesse.

11. Saint-François de Molitor. 